\title{
La inclusión educativa en la fiesta de la ciencia en España
}

\author{
A inclusão educativa na festa da ciência na Espanha \\ Educational inclusion at the science festival in Spain
}

\author{
José María Hernández Díaz \\ Profesor doctor em la Universidad de Salamanca, Salamanca, Espanha. \\ jmhd@usal.es \\ ORCID - http://orcid.org/0000-0001-7604-1544
}

Recebido em 5 de agosto 2020

Aprovado em 24 de agosto de 2020

Publicado em 17 de novembro de 2020

\section{RESUMEN}

La educación especial, hoy inclusiva, ha ocupado durante siglos una parte tangencial de los sistemas educativos de todo el mundo hasta nuestros días, incluido el de España. Era un sector al margen que quedaba fuera del circuito de la normalidad de los escolares y los estudiantes, que en el mejor de los casos comenzó a encontrar una atención especial, pero segregada. Los planteamientos de escuela integrada que prevalecen en muchas partes han apostado por un concepto socialmente más enriquecedor como es el de la educación inclusiva. Si observamos el problema desde la universidad, las nuevas categorías utilizadas para explicar la educación especial han tardado es ser aceptadas por la comunidad científica en los planos docente e investigador, si bien aquí se ha producido un salto espectacular en la consideración social y científica de la educación especial, al menos en España. En este artículo abordamos desde la universidad, y desde una lectura hermenéutica, el significado y visibilidad de las lecciones inaugurales en la llamada Fiesta de la Ciencia, que se pronuncian de forma cíclica, año tras año, en cada universidad española. Representan un excelente marco simbólico, un espacio privilegiado para observar la creciente presencia del paradigma científico de la inclusión educativa en el mapa universitario español. El escaso número de lecciones inaugurales pronunciadas en los últimos ochenta años con contenido próximo a la educación inclusiva expresan la debilidad del tema al contrastar con los grandes ámbitos de la ciencia, pero también son un motivo de esperanza dado que en los últimos años se observa una expresa emergencia del nuevo paradigma de la educación inclusiva, al menos en varias de las lecciones inaugurales pronunciadas en las universidades.

Palabras clave: Educación inclusiva; universidad; lección inaugural.

\section{RESUMO}

A educação especial, hoje inclusiva, ocupou durante séculos uma parte tangencial dos sistemas educativos de todo o mundo até nossos dias, inclusive o da Espanha. Era um setor à margem que ficava fora do circuito da normalidade dos escolares e dos estudantes, que na melhor das hipóteses começou a encontrar uma atenção especial, mas segregada. As abordagens de escola integrada que prevalecem em muitas partes apostaram por um conceito socialmente mais enriquecedor como é o da educação inclusiva. Se olharmos para o problema desde a universidade, as novas categorias para explicar a educação especial demoraram a ser aceites pela comunidade científica nos planos docente e investigador, embora aqui se tenha registado um salto espetacular na consideração social e científica da 
http://dx.doi.org/10.5902/1984686X49224

educação especial, pelo menos em Espanha. Neste artigo abordamos desde a universidade, e desde uma leitura hermenêutica, o significado e visibilidade das aulas inaugurais na chamada Festa da Ciência, que se pronunciam de forma cíclica, ano após ano, em cada universidade espanhola. Representam um excelente marco simbólico, um espaço privilegiado para observar a crescente presença do paradigma científico da inclusão educativa no mapa universitário espanhol. O escasso número de lições inaugurais pronunciadas nos últimos oitenta anos com conteúdo próximo da educação inclusiva expressam a debilidade do tema ao contrastar com os grandes âmbitos da ciência, mas também são um motivo de esperança dado que nos últimos anos se observa uma expressa emergência do novo paradigma da educação inclusiva, pelo menos em várias das lições inaugurais pronunciadas nas universidades.

Palavras-chave: Educação inclusiva; universidade; aula inaugural.

\section{ABSTRACT}

Special education, today inclusive, has occupied for centuries a tangential part of education systems around the world to this day, including that of Spain. It was a marginal sector that was outside the normal circuit of schoolchildren and students, who at best began to find special attention, but segregated. The integrated school approaches that prevail in many parts have opted for a more socially enriching concept such as inclusive education. If we look at the problem from the university, the new categories to explain the special education have taken is to be accepted by the scientific community at the teaching and research levels, although here there has been a spectacular leap in the social and scientific consideration of the special education, at least in Spain. In this paper we address from the university, and from a hermeneutic reading, the meaning and visibility of the inaugural lessons in the socalled Feast of Science, which are pronounced cyclically, year after year, in each Spanish university. They represent an excellent symbolic framework, a privileged space to observe the growing presence of the scientific paradigm of educational inclusion in the Spanish university map. The small number of inaugural lessons delivered in the last eighty years with content close to inclusive education express the weakness of the subject in contrast to the major fields of science, but they are also a reason for hope given that in recent years there has been an express emergence of the new paradigm of inclusive education, at least several of the inaugural lectures given at universities.

Keywords: Inclusive education; university; inaugural class.

\section{Introducción}

Sin remontarnos a una revisión histórica alargada en el tiempo pasado, hemos de confirmar que España ha sido un país pionero en el reconocimiento de los derechos educativos de las personas con dificultades de aprendizaje, movilidad, disminución de capacidades y de inserción ciudadana, y en la puesta en marcha de políticas sociales y formativas concretas encaminadas a resolver o mitigar todas esas circunstancias naturales o sobrevenidas a un sector de personas, que habían quedado aparcadas o expulsadas en la cuneta de la historia. Esto es muy visible desde 1970 en adelante, y en particular desde 
http://dx.doi.org/10.5902/1984686X49224

1990 y la conocida Declaración de Salamanca de 1994 sobre la educación especial, hasta nuestros días.

La pregunta que nos hacemos en esta ocasión procede también de la universidad, como institución de educación superior, y se centra en el grado de reflexión transversal que sobre el tema de la inclusión educativa realiza la propia institución de educación superior. Es cierto que la universidad en España, al menos en los últimos 30 años, ha establecido unidades de atención especializada para los alumnos que lo precisen y soliciten, y son servicios que funcionan muy bien. Pero no se trata ahora de estudiar eso. Es cierto también que son muchos los grupos de investigación reconocidos que abordan los problemas de la inclusión educativa, y antes denominada educación especial. Pero tampoco aquí nos centramos en ello.

Nos interesa ahora en este nuestro trabajo que presentamos reflexionar histórica y conceptualmente sobre el peso que el tema de la inclusión educativa llega a tener en un espacio privilegiado de gran simbolismo universitario y proyección social. Nos referimos a la Fiesta de la Ciencia, al acto y lección inaugural del curso académico que cada año se celebra ante autoridades científicas, académicas y civiles, y por supuesto estudiantes, profesores y personal de la universidad. Hablamos de las 87 universidades hoy existentes en España, principalmente públicas (50), sobre las privadas (37).

Hemos construido de forma laboriosa una detallada base de datos sobre las lecciones inaugurales pronunciadas en las universidades de España desde 1939 hasta 2019, lo que arroja una masa de 2165 unidades de análisis diferentes entre sí. Las hemos revisado de forma paciente durante varios meses, y así podemos obtener una imagen real sobre los intereses y paradigmas científicos que van aflorando (o desapareciendo) desde lo que los profesores senior de cada universidad van proponiendo a la comunidad científica. En ese mare magnum de procedencias intelectuales y académicas cabe todo, o casi todo. Por esto podemos preguntarnos también, ¿tiene algún peso significativo la inclusión educativa en los discursos que se pronuncian en los paraninfos universitarios de España, desde el alto estrado, y por los catedráticos más cualificados de su respectiva especialidad?

El resultado que nos arroja el estudio es poco amable para la educación inclusiva. Solamente aparecen siete Lecciones difundidas en la Fiesta de la Ciencia de las universidades españolas, con temas específicos de educación inclusiva. La esperanza es que parece ser un movimiento emergente del paradigma científico de la educación inclusiva, pues todos estos discursos se pronuncian ya en el siglo XXI, y se insinúan como 
http://dx.doi.org/10.5902/1984686X49224

un sendero a seguir por la comunidad científica. Eso es lo que analizamos en nuestro estudio, en su contenido científico y simbólico, donde aparecen cuestiones del currículum, el talento y la alta capacidad, el lenguaje y la inclusión educativa, la inclusión laboral por la educación.

\section{La Fiesta de la Ciencia en España}

La comunicación simbólica hoy es un instrumento arraigado en la vida cotidiana de las personas y de las instituciones, en el presente de una forma, y en siglos pasados con otras expresiones. Basta repasar mensajes icónicos en museos, en los textos escritos que maneja el historiador o el político de nuestro tiempo, en los diferentes medios de comunicación, en las redes sociales que con frecuencia nos atosigan de signos y símbolos como mecanismos de convicción a nuestra condición de consumidores o ciudadanos.

La universidad es desde su origen una institución compleja y cargada de simbolismo y rituales en el equilibrio de fuerzas y personas, y en particular del ejercicio del poder académico e influencia social y política. Por ello desde hace siglos la universidad utiliza determinados símbolos y actividades para mostrar a sus integrantes, a los visitantes y a quienes se colocan en proximidad de intereses y servicios quién es quién, y por qué razones. Ahí se sitúa el origen de la lectio medieval, precedente de nuestra lección inaugural, la que en estas páginas analizamos. Aquella ostentación intelectual pública nacida en Europa, el seno de las primeras universidades de la historia como modelo didáctico, era ante todo una forma de demostración pública de la valía científica de un profesor. En aquel ambiente y actos, cargados de ceremonial religioso, se producen esas manifestaciones de poder intelectual y símbólico que se integran en la oratoria académica. Así ocurre con determinadas fechas del calendario universitario, como es el caso de la lección inaugural del curso académico, siempre rodeado de protocolo, ropajes llamativos y una indudable reminiscencia del ceremonial religioso, de su liturgia.

Cuando se avanza en el proceso de secularización de las universidades, ya en el siglo XIX, y por indudable influencia del positivismo como corriente de pensamiento científico, son varios los catedráticos que intervienen como oradores de ese discurso inaugural los que comienzan a utilizar una denominación más laica y científica para referirse al acto de inauguración del curso académico. Comienza a utilizarse el sintagma "Fiesta de la Ciencia" para sustituir a la vulgar "inauguración del curso". Con ello estos catedráticos se refieren a los actos que cada universidad celebra de forma cíclica en sus inicios de curso, 
http://dx.doi.org/10.5902/1984686X49224

entre los cuales destaca por su solemnidad y prestigio la "Lección Inaugural". Durante todo el siglo XX y en el presente siglo XXI la celebración solemne de la inauguración del curso académico, de la Fiesta de la Ciencia, se convierte en uno de los actos de referencia de la identidad universitaria, de forma cíclica, año tras año, bien ritualizado y con gran poder simbólico de atracción para todos (DÍAZ, 2015).

La Fiesta de la Ciencia tiene un elevado contenido simbólico para toda la comunidad universitaria, para la ciudad que acoge la sede y para el conjunto de la sociedad. Para el orador u oradora encargado de pronunciar el discurso inaugural es un privilegio ser seleccionado, pues no cualquiera puede subir al estrado y mostrar ante auditorio tan selecto la defensa que hace de su disciplina científica, un comentario jocoso o serio de un problema del presente, la transmisión y efervescencia de determinados paradigmas científicos, y muchos más asuntos. Eso suele ocurrir una vez en la vida académica de un profesor, pero solo en la de algunos elegidos, muy pocos. Es un acto de confirmación del poder intelectual que se posee ante los oyentes que están presentes en el paraninfo de la universidad, 0 para aquéllos que leen la lección que se ha publicado, o quienes visualizan un reportaje por televisión o youtube.

La incógnita que nos interesa despejar aquí es relativa a la presencia o ausencia de la inclusión educativa entre los temas que se contemplan en las lecciones inaugurales de día tan señalado, en un lugar tan especial y por quienes son considerados académicos de excelencia por la comunidad científica y universitaria.

\section{La inclusión educativa en España, un proceso en marcha, y un reto por atender}

La pregunta parece pertinente para ser formulada porque interroga al mismo tiempo a la sociedad y a la universidad, así como sobre la función social y educativa que desempeña la universidad sobre determinadas demandas de la sociedad española, como es en este caso la educación inclusiva, lo que hasta hace bien poco se conocía con el nombre de la educación especial.

En lo que depende de la universidad, institución secularmente elitista, las personas con algún tipo de discapacidad encontraban infinitas dificultades para acceder a los estudios superiores y desarrollar su formación con normalidad y en plano de igualdad. Puede afirmarse que la universidad ha sido una institución ajena a las demandas de las personas diferentes, hasta hace bien pocos años. Podríamos admitir que se trataba de una posición socioeducativa situada en un plano semejante al del conjunto de la sociedad y del 
http://dx.doi.org/10.5902/1984686X49224

sistema educativo, en los que tales personas se encontraban marginadas y eran dejadas abandonadas en la cuneta de la vida, salvo la atención prestada por instituciones de beneficencia pública o de la iglesia a quienes se consideraban desvalidos, que en realidad sí lo eran.

En las universidades de España desde 1970 se ha avanzado de manera notoria en el tratamiento científico de la educación especial, en los planos docente e investigador, y preparando el debate y las políticas educativas específicas en el marco de la concepción comprehensiva de la educación que reconoce la Ley Orgánica General del Sistema Educativo (LOGSE) de 1990. Desde la aprobación de la Constitución de 1978, y la posterior implantación progresiva de políticas educativas de perfil socialdemócrata por parte del Partido Socialista Obrero Español (PSOE), la atención educativa a la diversidad, en todas sus expresiones, ha alcanzado niveles de éxito en muchos casos envidiables, tanto en el sistema educativo general, como en particular en las universidades, aunque aquí debamos introducir algunos matices más adelante (GONZÁLEZ, 2010). Una muestra de lo que indicamos queda recogida en la proclamación mundial de 1994, la denominada "Declaración de Salamanca" sobre la educación especial, que sienta nuevas bases de reflexión y actuación para muchas partes y países del mundo (ECHEITA et al. 2004).

De tal manera se ha avanzado en lo que se refiere a la presencia de la educación especial/educación inclusiva en las universidades, que se han instalado servicios generales de atención al estudiante y a su diversidad, programas de seguimiento específico y apoyo para estudiantes con discapacidad visual, auditiva, de movilidad y otros síndromes, que han de facilitar la inclusión y el éxito de este tipo de estudiantes en sus respectivos estudios universitarios. Además de la eliminación de barreras arquitectónicas y otros servicios de apoyo al estudiante, es imprescindible recordar aquí el peso creciente que en las Facultades de Educación (estudios de Magisterio y Pedagogía en particular) va alcanzando la mención educación especial/inclusiva en los programas de docencia que se ofertan en grados y másteres, así como la creciente presencia de estudiantes de doctorado interesados en investigar cuestiones relativas a algún aspecto conectado con la educación especial tradicional o la educación inclusiva. Por supuesto, crece en España el número de proyectos de investigación y especialistas investigadores en este campo, en ocasiones insertos en Institutos de Investigación especializados en la Educación Especial/Inclusiva.

Se ha avanzado mucho en las cuatro últimas décadas en la que se refiere a la presencia de nuevos estilos y paradigmas en el seno de la sociedad española y del sistema 
http://dx.doi.org/10.5902/1984686X49224

educativo en particular en relación a lo que se conecta con la educación especial/inclusiva. Pero esto no significa que todo ya esté resuelto, pues las incógnitas son todavía muy preocupantes (AINSCOW, 2011). Y cuando nos focalizamos en la universidad, que mira con otros ojos al conjunto de la ciencia, y a veces también a la sociedad, tenemos que expresar nuestras reservas. La universidad ha tardado en comenzar a incorporar este nuevo paradigma de la educación inclusiva en su organización y en las respuestas científicas que se le exigen como institución formadora e investigadora. El mejor ejemplo es el que aquí nos trae, como es la presencia de la educación especial/inclusiva en una actividad verdaderamente simbólica para una universidad, como es el discurso de la Fiesta de la Ciencia que se pronuncia año tras año en el paraninfo de la universidad, lugar sacralizado para el saber y la ciencia, si se nos permite utilizar esta expresión que es poco laica. Solamente en los últimos años comienza a ser visible la educación inclusiva en este espacio físico y mental de especial simbolismo, como vamos a ver.

\section{Metodología y fuentes de investigación utilizadas}

La metodología utilizada para este estudio se asienta en la lectura y rescate en bibliotecas, catálogos, bases bibliográficas documentales, repositorios, internet, youtube, de las lecciones inaugurales del curso académico pronunciadas en las universidades españolas de los últimos ochenta años (1939-2019). Advirtamos al lector que este número global de discursos inaugurales va en consonancia con el número de universidades existentes en España, y que éste ha evolucionado de manera casi exponencial desde 1969 hasta el presente. Si hasta esa fecha de hace cincuenta años podíamos hablar en España de la existencia de doce universidades públicas y cuatro confesionales católicas (Deusto y Comillas de los Jesuitas, Pontificia de Salamanca y Navarra del Opus Dei), en 2019 nos encontramos con 87 universidades (50 públicas y 37 privadas).

Hemos revisado nuestra base de datos sobre las lecciones inaugurales pronunciadas en las universidades españolas desde 1939 hasta 2019 (80 años de trayectoria institucional), y contabilizamos un cómputo total de 2.165 lecciones o unidades de análisis. Esa cifra está compuesta por las lecciones inaugurales pronunciadas cada año en las universidades españolas en el día señalado para el inicio del curso académico, normalmente una lección en cada una de las universidades, siendo escasísimo el número de las universidades que por alguna circunstancia no lo celebra, y en consecuencia no 
http://dx.doi.org/10.5902/1984686X49224

ofrece texto ni discurso publicado. La excepción la encontramos en alguna privada de perfil mercantil.

De ese conjunto de 2.165 intervenciones computadas leídas y escuchadas, mediante revisión paciente, hemos observado que son solamente siete las lecciones o discursos inaugurales que tienen como objetivo directo la presentación de algún asunto propio de la educación inclusiva. Es un número ciertamente muy reducido, más aún si lo comparamos con otros capítulos de la sociedad y la educación. Las lecciones son escritas y pronunciadas por cuatro hombres y tres mujeres, procedentes de Facultades de Educación, y todas son acogidas en las universidades, ya en el siglo XXI. Es revelador del panorama universitario y sociolingüístico español que de las siete lecciones dos se escriben y pronuncian en catalán (SOLER, 2015; LLANOS, 2015), una en gallego (SANTOMÉ, 2001) y cuatro en castellano (RODRÍGUEZ, 2016; DÍAZ, 2016; GASSET, 2016; FERNÁNDEZ, 2014). La distribución por universidades es muy dispersa por toda la geografía española (La Laguna, Salamanca, Universidad Autónoma de Madrid, UNED Madrid, Ramón Llull en Barcelona, Vic, y La Coruña) y no se repite en ninguna universidad la temática de la educación inclusiva en otras lecciones inaugurales pronunciadas. Esa distribución geográfica tan dispersa nos induce a pensar que el paradigma de la educación inclusiva se encuentra suficientemente reconocido en la cartografía científica y universitaria española.

\section{La inclusión educativa en la Fiesta de la Ciencia.}

Hemos optado por un repaso cronológico de cada una de estas lecciones inaugurales, lo que nos permitirá conocer y ubicar el punto de inflexión ascendente sobre la presencia de la educación inclusiva en las universidades españoles, a través del filtro que representa esta unidad de análisis que es la lección inaugural del curso académico en cada universidad donde se produjo.

En el contexto de una profunda reforma del sistema educativo español, representado en 1990 por la Ley Orgánica General del Sistema Educativo (LOGSE) emerge una posición entonces novedosa para el conjunto de la comunidad universitaria, que generalmente se había despreocupado de lo que sucede en el sistema educativo (SANTOMÉ, 2001). Desde la Universidad de la Coruña, anotamos que es el primer catedrático que en su lección inaugural propone a la universidad española una revisión del currículo de la escuela obligatoria desde planteamientos socioeducativos optimistas, pero a partir de supuestos organizativos del currículo mucho más justos y equitativos y socialmente más equilibrados. 
http://dx.doi.org/10.5902/1984686X49224

Y dentro de esa consideración incluye de forma explícita al sector de las personas diferentes, con capacidades distintas a las propias de la mal considerada homogeneidad normalizadora. Él es catedrático de Didáctica en aquella universidad gallega del norte de España, y apuesta en su discurso por un aula como lugar de inmensas posibilidades para todos los alumnos que la integran, y por una acción pedagógica para convertirla en un espacio de práctica de la libertad.

El aula de la escuela obligatoria debe ser promotora, nos dice, de una mayor igualdad social, mediante la práctica de un currículo realmente pleno de justicia social, la llamada justicia curricular. En este punto resulta decisiva la atención a las desigualdades de todo tipo que traen consigo los alumnos y alumnas que llegan a la institución escolar. Lo cual supone considerar la idoneidad pedagógica de los espacios, la formación adecuada de los profesores, el uso de metodologías diversificadas, la existencia de instalaciones deportivas y otras de apoyo a la orientación, el peso de las bibliotecas escolares y buenos laboratorios," las actividades y programas que busquen integrar e incluir a los alumnos que ofrezcan algún tipo de discapacidad y aspiren a construir con ellos expectativas optimistas acerca de su futuro" (SANTOMÉ, 2001, p. 63). En fín, primar de diferentes modos a quienes se dediquen más intensamente a este modelo de centro educativo inclusivo en todas sus dimensiones y aspectos.

Unos años más tarde en la Universidad Nacional de Educación a Distancia (UNEDMadrid) se pronuncia la lección inaugural del curso académico sobre el talento y las altas capacidades (FERNÁNDEZ, 2014). La autora de la extensa y documentada lección es catedrática de Pedagogía Diferencial de la UNED. Algunos epígrafes destacados del texto son: conceptualización de la alta capacidad, el retrato de los más capaces, chicos y chicas extremadamente capaces, alta capacidad y género.

Aunque se reconoce la dificultad de identificar con claridad los indicadores del talento (ASTUDILLO; CARLOS, 2015), con frecuencia reducido a un modelo de inteligencia, en muchos sistemas educativos del mundo occidental, y también en el de España, se ha comenzado a contemplar esta nueva realidad diferencial de los alumnos de altas capacidades en determinados sujetos de un centro educativo, que hasta hace muy poco tiempo pasaban desapercibidos o diluidos en la homogeneidad de un grupo, o presentaban conductas disociales, a veces muy extrañas y poco colaborativas, por no tener bien reconocidas sus capacidades y aptitudes en el proceso de socialización y de aprendizaje. Desde la Ley Orgánica de Educación de 2006 en España (LOE) se recoge de forma expresa 
http://dx.doi.org/10.5902/1984686X49224

esta nueva realidad, y se postulan respuestas desde las administraciones públicas y el magisterio en general, al menos en lo que se refiere al tramo de la educación obligatoria.

El texto de Jiménez Fernández se inscribe en este emergente movimiento en pro de los estudiantes de alta capacidad, y es concluyente cuando afirma, "La equidad justifica, en último término, la educación individualizada de los alumnos más capaces, y de todos los alumnos" (FERNÁNDEZ, 2014, p. 61)

Con gran proximidad temporal desde la universidad Ramón Llull de Barcelona se pronuncia otro discurso muy semejante en 2015 (SOLER, 2015), centrado en la conceptualización del talento y algunas de sus manifestaciones escolares diferenciales, lo que evidencia ser éste un tema de gran actualidad entre los integrantes de la comunidad pedagógica española.

En otra universidad catalana, la de Vic (LLANOS, 2015), la lección inaugural del curso académico 2015-16 se centra en estudiar el reto que representan los procesos de evaluación que emprenden los docentes de forma continuada, y el carácter inclusivo que deben adoptar, porque la evaluación integra un elevado sentido social. Esto es así porque, siendo un aula inclusiva la que acoge alumnos diferentes, los procesos de evaluación no pueden ser nunca homogéneos ni uniformes. Además, "una concepción inclusiva de la evaluación lleva consigo potenciar su función pedagógica" (LLANOS, 2015, p. 10), y de esa forma puede favorecerse una mayor inclusión social.

En 2016 se pronuncian en diferentes paraninfos universitarios españoles tres lecciones de contenido inclusivo. En la Universidad Autónoma de Madrid (IZUZQUIZA, 2016) se expone la exitosa experiencia inclusiva del programa Promentor, orientado hacia la inclusión laboral de determinados sectores de jóvenes socialmente discriminados y con dificultades de inserción en el mundo laboral. En la Universidad de La Laguna, en las Islas Canarias (RODRÍGUEZ, 2016), se analiza la importancia del lenguaje en la construcción de una educación y una sociedad más inclusivas, y en particular sobre su función para atenuar el fracaso escolar. Finalmente, en la Universidad de Salamanca (DÍAZ, 2016) al hacer una revisión de la paideia universitaria española, precisamente a través de las lecciones pronunciadas en las últimas décadas, se apuesta por una universidad que vaya configurando un modelo realmente inclusivo y pedagógico de formación universitaria acorde con los tiempos socioeducativos en que estamos ya ubicados. "Nuestra apuesta formativa, en una universidad pública como en la que creemos, es por una propuesta pedagógica integral (intelectual, estética, física, ética, comunitaria), y a la escucha de los 
http://dx.doi.org/10.5902/1984686X49224

problemas y grandes paradigmas que se van conformando en todo el mundo" (DÍAZ, 2016, p. 159).

\section{Discusión y conclusiones}

¿Qué reflexiones podemos destilar de este recorrido por la universidad española contemporánea en relación a la inclusión educativa, y desde lo que representa esta peculiar fuente de aproximación e información como son las lecciones inaugurales de la Fiesta de la Ciencia?

La primera apreciación que hemos de hacer al lector es que nuestra aportación busca ser una aproximación indirecta a la educación inclusiva en la universidad, analizada desde una fuente de información muy particular, aunque altamente representativa y simbólica para la vida universitaria, como es la lección inaugural del curso académico de cada año. Hemos abordado desde una duración histórica media de ochenta años (1939-2019) la presencia y ausencia de la educación inclusiva en un territorio completamente inusual para el estudio de la educación especial como es el discurso universitario de alto nivel. Pensemos que quienes pronuncian la lección inaugural en una universidad cualquiera son catedráticos senior en una muy diversificada tipología de saberes y ciencias (Humanidades, Ciencias Sociales, Ciencias Experimentales, Ciencias de la Vida, Tecnológicas), elegidos por el equipo de gobierno de la universidad, y ellos suelen hablar y escribir de lo que saben. Esto explica que la gran mayoría de quienes hablan desde el estrado del paraninfo no pertenezcan a las Ciencias de la Educación, y por tanto las oportunidades de que se vean reflejados los temas educativos en esas lecciones es escasa en su conjunto y muy limitada de planteamientos, en el mejor de los casos

Por otra parte, el peso del ámbito de la educación en el conjunto de una universidad española, y por tanto de sus catedráticos, es menor y más reciente, si lo comparamos con otras especialidades que a veces tienen una trayectoria de varios siglos (casos del derecho, la filosofía o la medicina, entre otros). En consecuencia, es mucho más probable que nos encontremos en la Fiesta de la Ciencia con lecciones inaugurales que procedan de tales territorios científicos antes que en el de la educación. Pensemos, además, que la Pedagogía alcanza en España rango universitario en 1932, cuando se crea en Madrid la primera Facultad, y que la Psicología solo alcanza a ser rama universitaria oficial y reconocida a partir de 1973. Si trasladamos este balance al proceso de construcción de las profesiones de catedráticos de Pedagogía y de Psicología podemos deducir el retraso de 
http://dx.doi.org/10.5902/1984686X49224

llegada a los máximos niveles del escalafón académico, los catedráticos, de los profesores procedentes de estas especialidades científicas. Por todo ello, parece lógico comprender que el ámbito de la educación solo haya llegado recientemente a los máximos espacios de influencia y de visibilidad del saber y la ciencia en la universidad, uno de los cuales es sin duda la lección inaugural en la Fiesta de la Ciencia.

Dentro del espacio que podemos considerar como representativo de las Ciencias de la Educación hemos de añadir algunos matices. Así, que el ámbito específico de la educación especial/inclusiva es uno de los más recientes en su incorporación al mapa de los ámbitos disciplinares que conforman las Ciencias de la Educación, debiendo competir en el día a día con otros de la Didáctica, la Filosofía de la Educación, la Sociología de la Educación, la Psicología de la Educación, la Educación Comparada, la Historia de la Educación, la Educación Social, la Organización Educativa, las Didácticas de especialidad (matemática, ciencias experimentales, ciencias sociales, educación física, plástica, musical) entre los más influyentes. Podríamos afirmar, que en la tradición de la pedagogía contemporánea la educación especial/inclusiva es una recién llegada, por las razones ya explicadas de la tardía presencia que los sistemas educativos de todo el mundo, incluido el de España, han concedido a la educación inclusiva, siendo así solamente desde hace no más de cincuenta años en el mejor de los casos. Eso explica también que sean muy escasos los catedráticos senior, especialistas en este campo de la educación especial/inclusiva, que estén en condiciones de ser nominados por las autoridades universitarias para pronunciar esa simbólica lección inaugural del curso académico de una universidad.

La llegada de la educación inclusiva a la universidad, tal como expresan las lecciones inaugurales que analizamos, se produce con retraso de varias décadas respecto a lo que ya se viene fraguando en el sistema educativo obligatorio y precedente a la universidad. Lo que significa que si bien es cierto que la universidad con frecuencia abre espacios nuevos a la sociedad y a su sistema educativo, no es menos cierto también que a veces la universidad es una institución que se mueve con lentitud para percibir y responder a los problemas que se plantean en la sociedad, en este caso los propios de la educación especial/inclusiva. Es muy propio de la institución universitaria el moverse en esta dialéctica, que nunca es un movimiento uniforme ni se produce en una sola dirección.

Merece la pena observar a través de estos discursos cómo se ha enriquecido el concepto de educación de especial/inclusiva al incluir la educación y atención de los 
http://dx.doi.org/10.5902/1984686X49224

alumnos de altas capacidades, que tampoco hasta hace bien pocos años merecían más atención, y que a veces representaban un problema real en las aulas de un centro educativo. No es casual que dos de las siete lecciones se centren precisamente en el estudio del talento y la alta capacidad desde planteamientos inclusivos.

Con la educación especial/inclusiva incorporada a la docencia e investigación universitarias, a la formación de profesionales para el sistema educativo y los avances científicos en ese ámbito de tanto interés social y educativo, se está produciendo en la universidad española (probablemente en la mundial también) la llegada y reconocimiento de un nuevo paradigma científico, de tipo transversal, como es el de la educación inclusiva. Pero ya sabemos que para que los paradigmas se vean reconocidos y fortalecidos en la ciencia se precisa de un trasiego, de un tiempo histórico y de mucha práctica social y contrastada. Lo que nos indica la todavía incipiente presencia del paradigma de la educación inclusiva entre el selecto cupo de los catedráticos elegidos para pronunciar su lección en el día de la Fiesta de la Ciencia es que estamos asistiendo a un movimiento científico en las Ciencias de la Educación, representado por la educación inclusiva, que gana peso en su seno, pero que también va ensanchando la percepción social de este campo científico novedoso para la gran mayoría de la comunidad científica y universitaria. La Fiesta de la Ciencia, y sus reveladoras lecciones magistrales, se convierten así en un excelente indicador de los movimientos científicos novedosos que se van produciendo en las diferentes especialidades, así como en un espejo público del máximo simbolismo social y universitario, y desde luego científico. Ahora ya sabemos que en el siglo XXI el paradigma científico de la educación inclusiva ha llegado a la sociedad y a la universidad, ha logrado visibilidad social y reconocimiento científico, y por supuesto que ha de fortalecerse en las próximas décadas.

En este trabajo nos servimos de fuentes originales, las lecciones inaugurales, nunca hasta ahora utilizadas para fines semejantes en lo que se refiere a la educación especial/jinclusiva, que nos permiten aplicar criterios hermenéuticos actuales, por el peso que lo simbólico tiene en la vida de las instituciones y las personas. Por eso decimos que el paraninfo de la universidad es el lugar por excelencia para ofrecer a la comunidad universitaria, y desde ahí al conjunto de la sociedad, nuevos puntos de vista sobre la educación y la educación inclusiva en concreto, en el marco general de lo que se piensa sobre el sistema educativo en su conjunto, del que la universidad también forma parte, o debiera hacerlo en nuestra opinión. Las palabras y las cosas, los espacios también, nos 
http://dx.doi.org/10.5902/1984686X49224

podría decir Michel Foucault, muestran a los hombres y mujeres de todo tiempo y lugar nuevas formas de relación y de comprensión del mundo, más allá de lo explícito y obvio. Por esto quedamos invitados a pensar y observar críticamente las expresiones orales y escritas, pero también los gestos, movimientos, ropajes, música de acompañamiento, ambiente que expresa un acto social, en este caso en la universidad, y en concreto en su paraninfo en un día tan especial como es el día de la Fiesta de la Ciencia, en el que la educación inclusiva comienza a alcanzar reconocimiento científico de forma simbólica y presencia social.

\section{Referências}

AINSCOW, Mel. Responding to the challenge of equity within education systems, Aula. Revista de Pedagogía de la Universidad de Salamanca. 17, 73-87. 2011.

ASTUDILLO, María Teresa González; CARLOS, Fernando Sergio Domingues. ¿Existen indicadores para identificar el talento? Aula. Revista de Pedagogía de la Universidad de Salamanca. 21, 21-32. 2015.

DÍAZ, José María Hernández. "El discurso pedagógico en la Fiesta de la Ciencia" en la universidad deseada: España, 1900-1936”. Historia de la Educación. 34, p. 103-138. 2015.

DÍAZ, José María Hernández. La paideia universitaria en la Fiesta de la Ciencia. Lección inaugural del curso académico 2016-17 en la Universidad de Salamanca. Salamanca: Universidad de Salamanca. 2016.

ECHEITA, Gerardo; VERDUGO, Miguel Ángel. La Declaración de Salamanca sobre Necesidades Educativas Especiales 10 años después. Valoración y prospectiva. Salamanca: INICO. 2004.

FERNÁNDEZ, Carmen Jiménez. El desarrollo del talento: educación y alta capacidad. Lección inaugural del curso académico 2014-15 en la UNED. Madrid: UNED. 2014.

GASSET, Dolores Izuzquiza. El camino hacia la inclusión laboral en la sociedad del siglo XXI. Promentor: en la vanguardia de la universidad de excelencia. Lección inaugural del curso 2016-17 en la Universidad Autónoma de Madrid. Madrid: UAM. 2016.

GONZÁLEZ, Carmen Ortiz. De la Pedagogía Terapéutica a la Educación Esprecial, desde una óptica profesional. In: DÍAZ, José María Hernández (Ed.). Cien años de Pedagogía en España. Valladolid, Castilla Ediciones. 2010. pp. 111-123.

LLANOS, Milagros Naranjo. L’avaluació com a eina d'inclusió educativa i social. Lliçó inaugural del curs académic 2015-16 en la Universitat de Vic. Vic, Universitat de Vic, pp. 17. Digital. 2015. 
RODRÍGUEZ, Víctor Acosta. Lenguaje e inclusión educativa. Reflexiones para atenuar el fracaso escolar. Lección inaugural del curso académico 2016-17 en la Universidad de La Laguna. La Laguna, Universidad de La Laguna. 2016.

SANTOMÉ, Xurxo Torres. Un currículo optimista fronte a desmemoria e o fatalismo. Lección inaugural na solemne apertura do curso académico 2001-02 da Universidade da Coruña. A Coruña, Universidade da Coruña. 2001.

SOLER, Josep María Lozano. Notes (im)pertinentes sobre el talent. Lliçó inaugural del curs académic 2015-16 en la Universitat Ramón Llull. Barcelona, Universitat Ramón Llull. 2015.

\section{Corespondencia}

José María Hernández Díaz - Universidade de Salamanca, Facultad de Educación, Paseo de Canalejas, 169, Salamanca - Espanha.

CEP: 37008

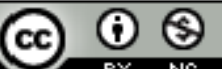

4.0 International (CC BY-NC 4.0) 